\title{
Biosensors application for the life systems quality in a smart city
}

\author{
Viktoriia Zaripova ${ }^{1}$, Irirna Petrova $^{1}$, and Yuliya Lezhnina ${ }^{1 *}$ \\ ${ }^{1}$ Astrakhan state university of architecture and civil engineering, CAD\&M department, Astrakhan, \\ Russia
}

\begin{abstract}
The article shows that the smart city management systems requires continuous monitoring of environmental parameters (air, soil and water quality, temperature, humidity, toxic impurities, etc.) and the placement of a large number of different sensors, among which biosensors occupy a leading position. The study analyzed patent documents for leading countries of the world (Great Britain, France, Japan, Germany, USA, Russia) and it showed a steady increase in the number of patents on biosensors. The reasons for the growth and continuous expansion of the scope of biosensors are: cost reduction, increased durability, speed, reliability, accuracy and sensitivity; increase in their functionality. The article presents a classification of biosensors indicating the percentage of patents by type of transducer and the number of patents by bioselective elements, as well as a description of an automated system for the synthesis of new biosensor designs based on the energy-information method. Paper is given the resulting solution can be used as a skeletal structure to create new biosensors, or improved using the knowledge base on techniques for improving the technical and operational characteristics of physical and technical effects.
\end{abstract}

\section{Introduction}

In $2010,50 \%$ of the world's population was already living in cities. This figure is projected to rise to $75 \%$ by 2050 . [1] This will make urbanization one of the most transformative trends of the twenty-first century. Information technology is an active part of the urban infrastructure now. At the beginning of the XXI century there was a term Smart City ("Smart city"), but still there is no single definition of this term.

In 2015, the United Nations economic Commission for Europe (UNECE) proposed the following definition of a smart city: "a Smart city is an innovative city that uses information and communication technologies (ICT) and other means to improve the quality of life, the efficiency of urban operations and services and competitiveness, while meeting the needs of present and future generations with regard to economic, social, environmental and cultural aspects" [2].

The growth of the world's urban population leads to even greater economic inequality. Resource scarcity, climate change and environmental pollution will become even more

\footnotetext{
*Corresponding author: leninau@mal.ru
} 
serious problems at the global level [3]. Cities will be particularly vulnerable because of the high concentration of population, which increases the pressure on resources such as land, space, clean water and energy [4].

The issues of effective management of large cities and ensuring the vital functions of the urban environment (transport, security, water supply, energy, etc.), necessary to meet the needs of citizens, are becoming even more relevant. The solution to these issues is possible only on the basis of information and communication technologies (ICT). The use of ICTs in smart cities will reduce energy consumption, water and $\mathrm{CO}_{2}$ emissions. This will improve the use of the existing infrastructure of the city, provide new services to citizens and improve the quality of life [5].

The system architecture of the infrastructure of smart cities is connected with several latest technologies: information and communication (ICT), the Internet of Things (IoT), wireless sensor networks (WSN), etc. The infrastructure of a smart city can be represented in several layers, as shown in Fig. 1.

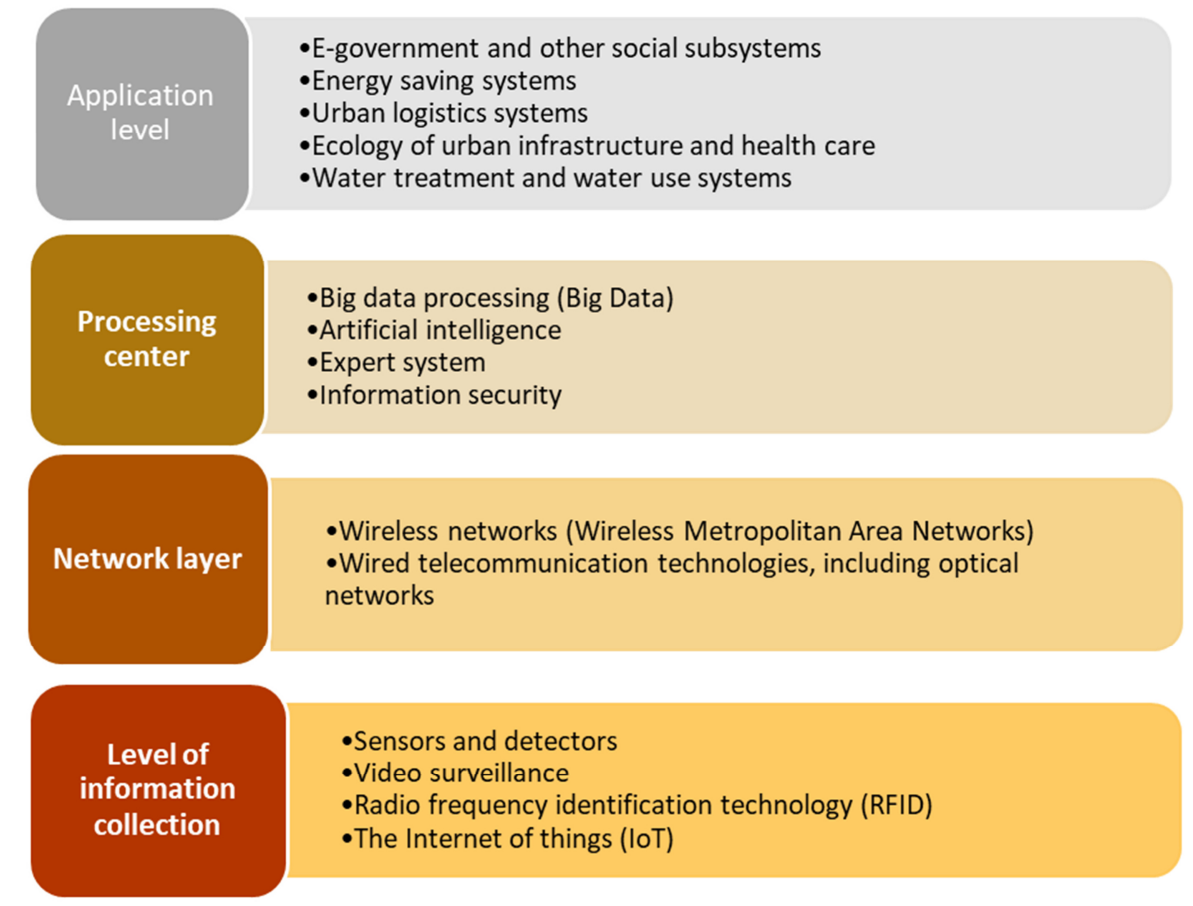

Fig.1. System architecture of smart city infrastructure.

On the first layer, the sensors collect data over the network and send them for processing to the central processing center, where expert control decisions are formulated and big data is processed (BigData), which are then implemented at the application level $[6,7,8]$.

\section{Integrated sensor systems for smart cities}

Sensors are an essential component of any intelligent control system. Complex urban control systems require many sensors of different operating principles. The development of new real-time detection and monitoring devices plays an important role in protecting human and environmental resources from harmful substances. This article discusses the 
design of sensors for some specific aspects of smart cities, such as environmental monitoring, water resources, air quality and environmental safety.

Among the many different types of sensors, biosensors are the most popular applications for real-time monitoring of the environment, water and air quality. The paper $[9,10]$ describes the application of biosensors in the design of smart city management systems.

\subsection{Biosensors}

In 1997, the International Union of theoretical and applied chemistry (IUPAC) introduced the definition of a biosensor as a device consisting of a transducer and an immobilized biological element [9].

A biosensor is an analytical system containing biological material (enzymes, cells, antibodies, antigens, receptors, DNA fragments) that is in direct contact or embedded in a physico-chemical sensor. A generalized scheme of construction of biosensor devices isshown in figure 2.

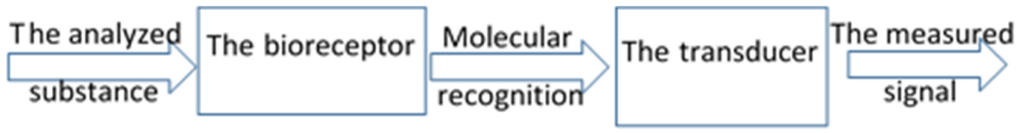

Fig.2. Generalized biosensor circuitry.

Biosensors consist of 2 parts:

1. Biological sensing element-bioselective element. It is a complex of biological molecules in which physical and chemical processes convert the properties of the environment under study into a measurable signal (electrical, optical, mechanical, thermal, etc.). For example: microorganisms, organelles, cell receptors, enzymes, antibodies, nucleic acids, etc.

2. Transducer-converts a signal that appears as a result of interaction of an analyte with a bioselective element into another signal that is easier to measure. Various physical and chemical principles of action are used: optical, piezoelectric, electrochemical and others.

Typically, a biosensor is designed to generate a digital electrical signal proportional to the concentration of a particular chemical compound or series of compounds.

The authors analyzed patent documentation using information resources of the world intellectual property organization (http://patentscope.wipo.int/). the Subject of the search were biosensors and their varieties. The search for patents was carried out in the following applicant countries: UK, France, Japan, Germany, USA, Russia, as well as the International patent system (PCT) and the European patent office.

As a result of patent analysis on databases of the world intellectual property organization (http://patentscope.wipo.int/) 99,781 patents were found for the period 2008 2019 on the request "biosensor" in English and only 844 patents on the request "biosensor" in Russian (of which 557 patents are registered in Russia). The dynamics of changes in the number of registered patents by year is shown in Fig.3. Analysis of this chart shows that over the past 10 years, interest in this topic in the world is constant and remains quite high. 


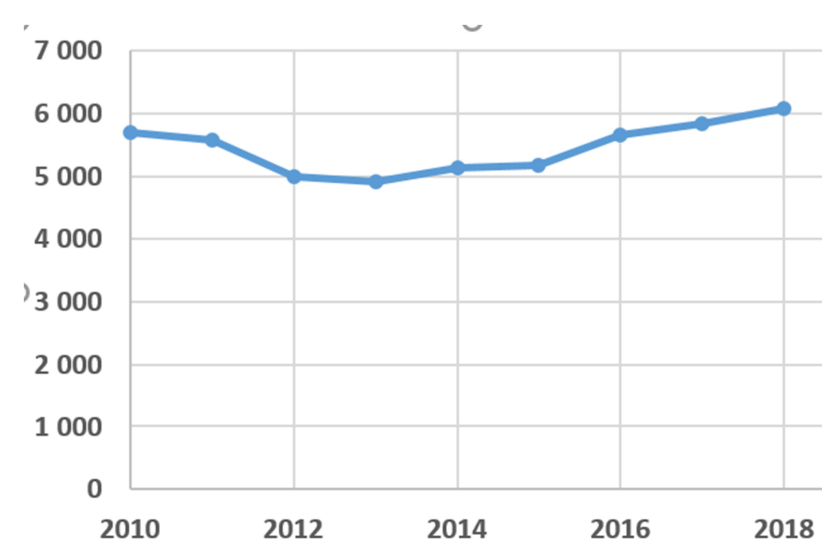

Fig.3. Dynamics of changes in the number of biosensor patents.

The main number of patents issued both in Russia and abroad belongs to the class G01N of the international patent classification (IPC) - research or analysis of materials by determining their chemical or physical properties (42.4\%).

The reasons for the growth and continuous expansion of the scope of biosensors are: reducing cost, increasing durability, speed, reliability, accuracy and sensitivity; increasing their functionality. Today biosensors are widely used in environmental quality control, water, air monitoring, toxicology.

\subsection{Classification of biosensors by the principle of action}

A large number of papers are devoted to the classification of biosensors. [10,11,12]. Figure 4 shows the classification of biosensors considered in this paper, indicating the percentage of patents by type of transducer and the number of patents by test objects.

\subsection{Classification by bioselective element (by test objects)}

Immunosensors (antibodies) use immunoglobulins as a biochemical receptor - these are protective proteins that are secreted by the body's immune system in response to exposure to foreign biological compounds (antigens). Antibodies are ideal elements of biological recognition that provide high selectivity and sensitivity. Used to detect environmental pollution, traces of narcotic substances and toxicology [13].

Enzyme sensors suggest biological preparations (homogeneous to microbial cultures or tissues) or pure enzyme preparations that exhibit a certain biological activity and generate a signal in proportion to the analyte concentration. This signal can occur as a result of changes in the concentration of protons, the release or absorption of gases such as ammonia or oxygen, the emission of light, the absorption or reflection of light, the generation of heat, etc. [14]

Microbial sensors involve microorganisms that are capable of carrying out the conversion of a specific substance using enzymes. They differ from enzyme sensors in that during the transformation of the substrate, a combination of enzymes can be used, and not just one enzyme [15].

The use of slices of biological tissues eliminates the disadvantages inherent in enzyme biosensors. In this case, there is no need to obtain and purify enzymes. These biosensors have low selectivity and greater RTT [18].

DNA sensors include nucleic acids (DNA) as a biochemical component. Biosensors based on supramolecular cell structures are in an intermediate position between DNA 
sensors, enzyme and microbial sensors, since they are based on intracellular structures, which have a very complex hierarchical structure [19].

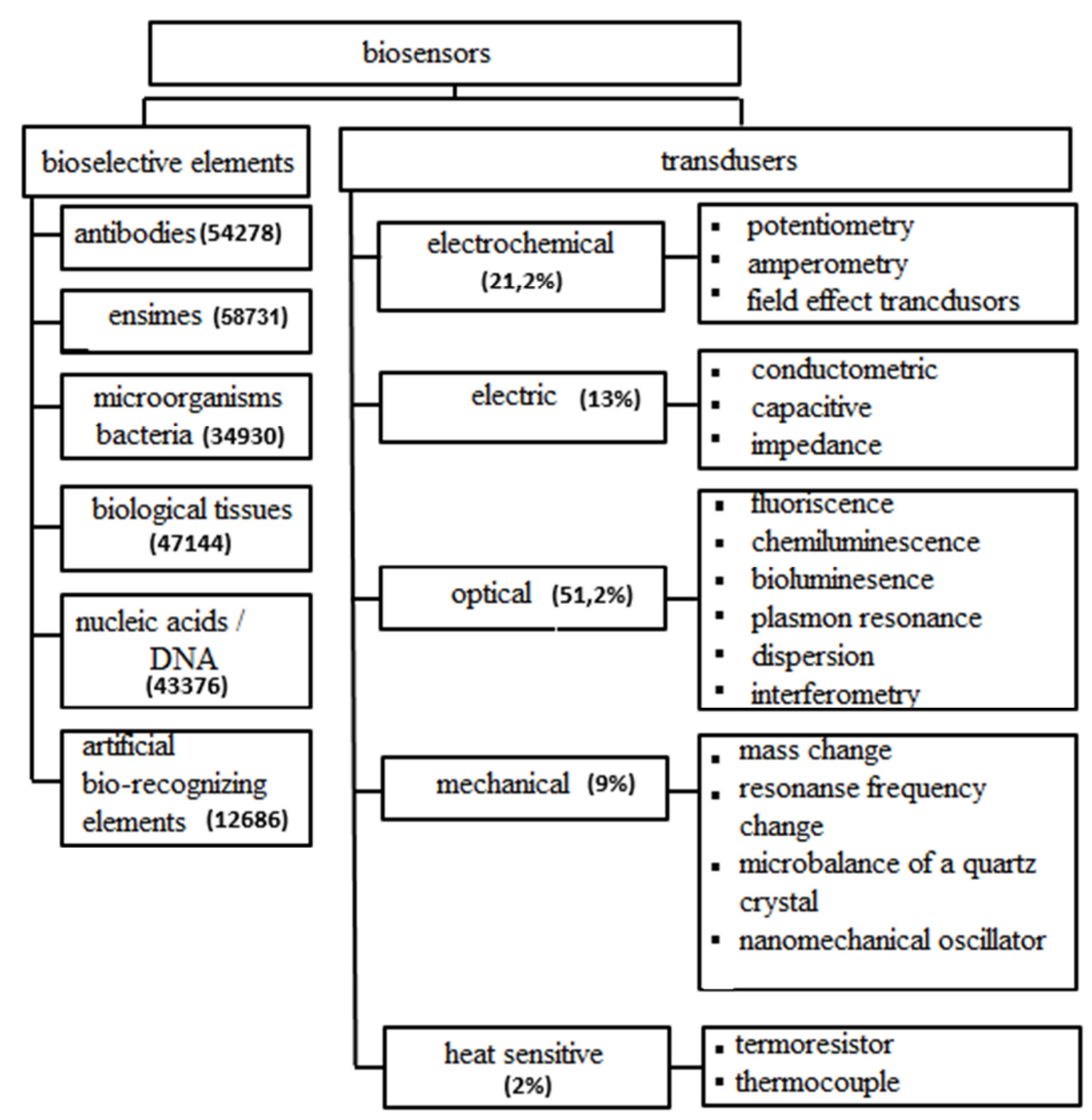

Fig. 4. Classification of biosensors.

Artificial bio-recognizing elements - aptamers, peptides, polymers obtained by molecular printing or artificial associations of microorganisms, activated sludge, etc. [20]. For example, a mixture of two or more identified strains is used in a bio-recognizing element. This allows you to stabilize the sensor for a long period and expand the range of recognized impurities.

\subsection{The classification of biosensors by type of transducer is presented below}

Electrochemical transducers are based on the measurement of electrical quantities (current or voltage) that change during the interaction between the receptor and analyte. A potentiometric sensor generates a signal as a potential difference between a working electrode and a reference electrode immobilized in a semipermeable membrane. In amperometric biosensors, the concentration of the component being determined is judged by the change in the electric current, and not the potential. In biosensors based on fieldeffect transistors (MOS transistors), the transistor gate acts as an indicator electrode. 
Electrical transducers are based on measuring the electrical parameters of a medium (resistance, capacitance or impedance - complex electrical resistance). Conductivity sensors measure the conductivity of the solution during the course of the analytical reaction. Conductivity biosensors are widely used to monitor the environment, in particular air [21]. Impedance sensors are based on measuring resistance in an electrochemical cell or on recording changes in resistance with varying voltammetric characteristics [22]. These sensors are promising for the detection of foodborne pathogenic bacteria, have high sensitivity and speed. Capacitive biosensors measure changes in the dielectric properties and / or thickness of the dielectric layer at the electrolyte/electrode interface. Capacitive biosensors are used to detect proteins, nucleotides, heavy metals, saccharides, small organic molecules and microbial cells [23].

Optical biosensors are based on the phenomena of absorption, reflection or luminescence, as well as on the effect of surface plasmon resonance [24,25]. Fiber-optic cable biosensors have the following advantages: noise immunity, low noise level. They are used to detect pathogens, pesticide and drug residues, heavy metals and other toxic substances in environmental monitoring.

Mechanical biosensors are based on several phenomena. A piezoelectric quartz biosensor is a resonator with electrodes coated with receptor molecules. An analytical signal is the change in the oscillation frequency of the resonator with an increase in the mass of the receptor layer as a result of its interaction with the analyte. Piezoelectric quartz biosensors have high sensitivity, speed and selectivity [26]. Nanoscale mechanical biosensors in which the deformation or vibration of a moving element changes upon molecular adsorption. This subcategory provides superior mass resolution - down to one molecule. Compared to quartz resonators, the size of the nanomechanical biosensor allows one to provide mass resolution on the face of individual atoms [27].

Thermosensitive biosensors are based on the effect of heat release during a chemical reaction. These biosensors have several advantages compared to amperometric and optical methods (easy manufacturing, less sophisticated signal detection systems) [28]. Thermocouples, thermistors are used as transducers [29]

In table 1. comparative characteristics and applications of biosensors in smart city control systems are shown.

Table 1. Fields of application of biosensors in smart city control systems.

\begin{tabular}{|c|c|c|}
\hline Biosensor type & $\begin{array}{c}\text { Application in smart city } \\
\text { management systems }\end{array}$ & Biosensor Specifications \\
\hline $\begin{array}{c}\text { Electrochemical } \\
\text { biosensors }\end{array}$ & $\begin{array}{c}\text { Environmental monitoring, } \\
\text { wastewater }\end{array}$ & $\begin{array}{c}\text { High sensitivity } \\
\text { Wide linear range }\end{array}$ \\
\hline Electric biosensors & Environmental monitoring & High sensitivity and speed \\
\hline Optical biosensors & $\begin{array}{c}\text { Detection of a chemical } \\
\text { reagent in soil, air or water. } \\
\text { Toxicology. }\end{array}$ & $\begin{array}{c}\text { Low power and low cost } \\
\text { Selectivity to analyte } \\
\text { High response time }\end{array}$ \\
\hline Mechanical biosensors & $\begin{array}{c}\text { Environmental Monitoring, } \\
\text { Food }\end{array}$ & $\begin{array}{c}\text { Inexpensive, compact and } \\
\text { portable. } \\
\text { High sensitivity, speed and } \\
\text { selectivity }\end{array}$ \\
\hline $\begin{array}{c}\text { Thermosensitive } \\
\text { biosensors }\end{array}$ & Environmental monitoring & $\begin{array}{c}\text { Easy fabrication, less } \\
\text { sophisticated signal detection } \\
\text { systems, versatility, stability }\end{array}$ \\
\hline
\end{tabular}




\section{Designing biosensors for smart city}

Theoretically, any bioselective element can be combined with any of the possible transducers, which are a combination of several physical and technical effects. Therefore, a wide variety of different types of biosensors is possible. In the works of the authors [30,31] A description is given of an automated system for the synthesis of new biosensor designs based on the energy-information method (EIM).

Figure 5 shows a data flow diagram (DFD model) of a biosensor conceptual design system. The system interface allows the user to enter a design assignment in the form of the following data set: substance to be recognized; type of output signal of the test object; transformation chain length; vector of values of the basic set of operational characteristics of solutions; the weight vector of these characteristics in points from 1 to 10 , where 1 is the least significant, 10 is the most significant characteristic.

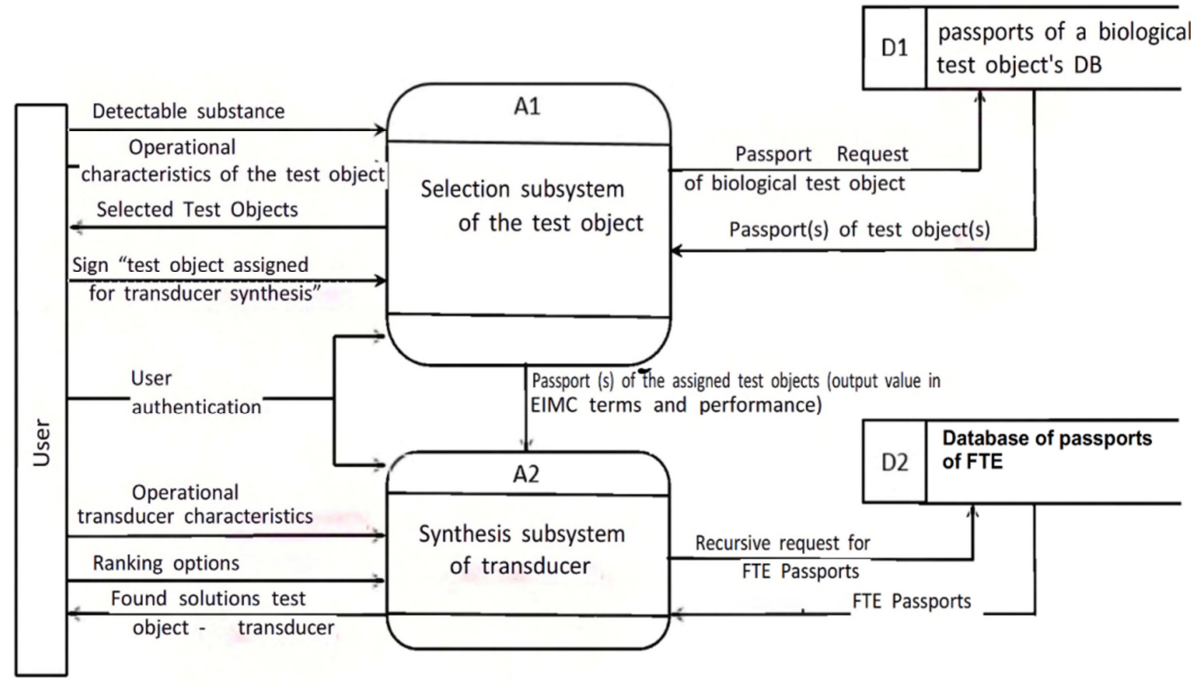

Fig. 5. Data flow diagram of an automated system. logic:

The bioselective element selection subsystem implements the following user interaction

1. Request to the database of passports of bioselective elements based on data on a recognized substance and its operational characteristics. An example of a bioselective element passport is shown in Fig. 6

2. Selection from the database of bioselective elements of a finite set of objects that satisfy the given conditions and have an output value compatible with the input value of the physical and technical effect (PTE) from the database of TFE passports. The output value of the bioselective element is described in terms of EIM, which allows the use of the existing volume of PTE passports [30, 31].

3. Providing the user with information about selected bioselective elements, ranked taking into account the user-specified significance of operational characteristics.

Based on the data provided by the bioselective element selection subsystem, the user can select one of the many objects or start the search for solution options for the entire set of objects, passing further control over the selection of the solution to the transducer synthesis subsystem. 


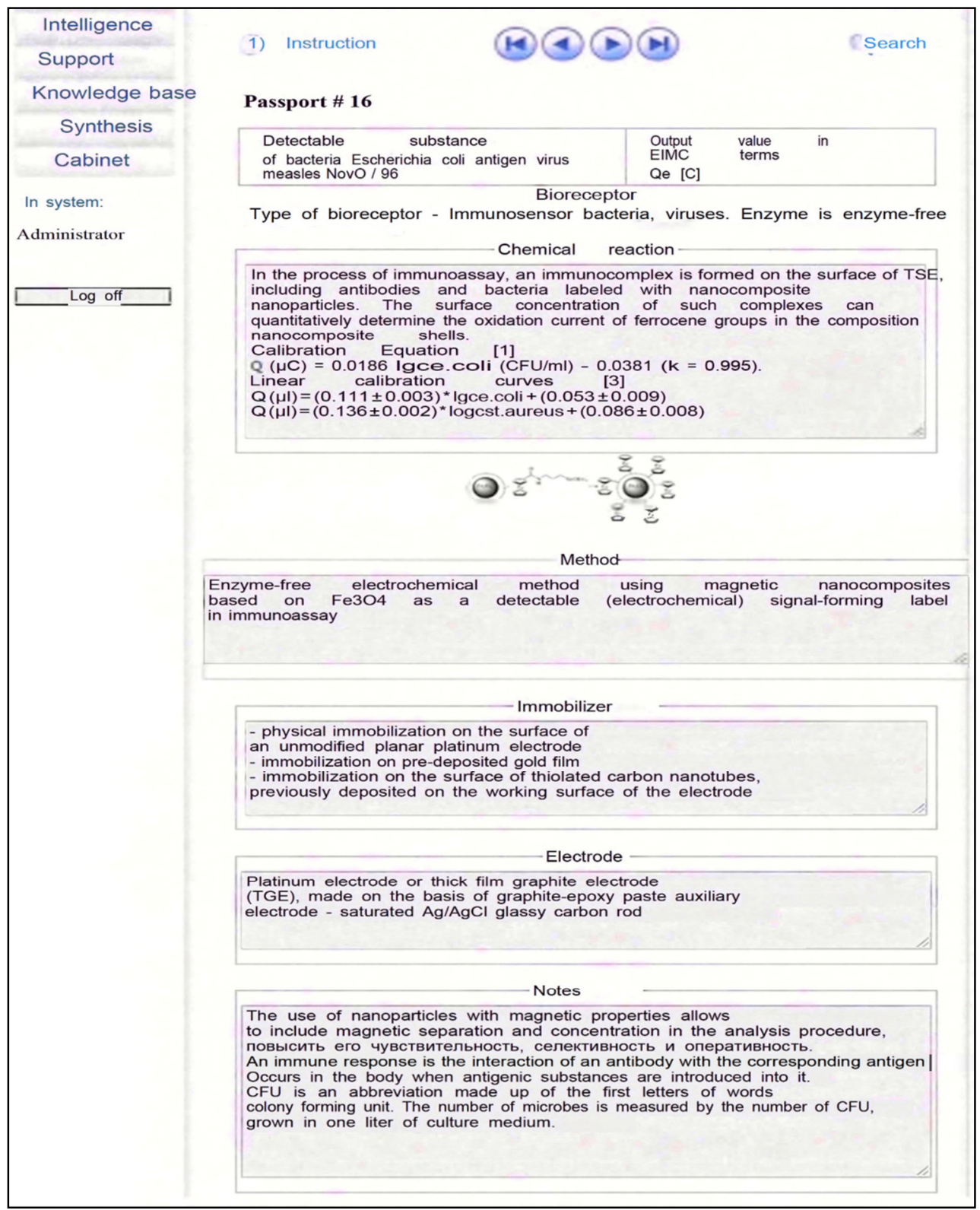

Fig. 6. Example passport bioselective element.

The transducer synthesis subsystem implements the following user interaction logic:

1. Recursive query to the database of PTE passports for constructing a chain of transformations, starting from a given output value of a bioselective element, which is an input to a chain of transformations, and ending with the required output value of a transducer. This takes into account the restrictions set by the user along the length of the chain, as well as the coincidence of the ranges and natures of the input and output values of the effects in the chain, since in the case of mismatch between the ranges and the physical nature of the value, the signal cannot be converted.

2. Calculation of the vector of values of the operational characteristics of the final solution based on the set of values of the characteristics of the elements of the 
transformation chain. The average values described for a typical structural and technical implementation of this physical effect are used as the values of the characteristics of the PTFE.

3. Ranking of the obtained solutions by the vector of characteristics taking into account the weights of the characteristics specified by the user.

4. Providing the user with information about the decision in the form of a set of passports - a test object and the associated chain of PTEs.

As a result, the resulting solution can be used as a skeletal structure to create new biosensors, or improved using the knowledge base on techniques for improving the technical and operational characteristics of physical and technical effects.

\section{Conclusion}

The smart city management system requires continuous monitoring of environmental parameters (air, soil and water quality, temperature, humidity, toxic impurities, etc.) and the placement of a large number of different sensors, among which biosensors occupy a leading position.

Analysis of patent documents for leading countries of the world (Great Britain, France, Japan, Germany, USA, Russia) showed a steady increase in the number of patents on biosensors. The reasons for the growth and continuous expansion of the scope of biosensors are: cost reduction, increased durability, speed, reliability, accuracy and sensitivity; increase their functionality.

The article presents a classification of biosensors indicating the percentage of patents by type of transducer and the number of patents by bioselective elements, as well as a description of an automated system for the synthesis of new biosensor designs based on the energy-information method (EIM). The system can be used in scientific research and in experimental design work in order to reduce the complexity of the process of conceptual design of biosensors. Using this system will reduce design time by an average of $5-10 \%$.

\section{Referenses}

1. UN-HABITAT. 2016b. World Cities Report 2016. Urbanization and development: Emerging futures, http://wcr.unhabitat.org/main-report/

2. V. Akberdina, L. Pushkareva, 4th International Conference on Social, Business, and Academic Leadership (ICSBAL 2019) (Atlantis Press 2019).

3. G. P. Hancke, B. Carvalho e Silva, G. P., Hancke Jr., Sens., 13(1), 393-425 (2013) doi:10.3390/s130100393.

4. Y. Li, Y. Lin, S. Geertman, Proceedings of the 14th Int. Conference on Computers in Urban Planning and Urban Management I Cambridge, MA USA, 7-10 (2015).

5. C. Harrison, A. Donnelly, Proceedings of the 55'h Annual Meeting of the ISSSI Hull, 55(1), 1-15, (2011).

6. K. Su, J. Li, H. Fu, 2011 International Conference on Electronics, Communications and Control (ICECC), Ningbo, 1028-1031 (2011) doi:10.1109/ICECC.2011.6066743

7. M. Jaradat, M. Jarrah, A. Bousselham, Y. Jararweh, M. Al-Ayyoub, Comp. Sci. (56) 592-597 (2015) doi:10.1016/j.procs.2015.07.250

8. Bacic, Zeljko, T. Jogun, I. Majić, Tech. gaz. 25 277-284 doi:10.17559/TV20160620125732. 
9. S. Shamsir, I. Mahbub, S. K. Islam and A. Rahman, IEEE 60th International Midwest Symposium on Circuits and Systems (MWSCAS), 1150-1153 (2017) doi: 10.1109/MWSCAS.2017.8053132

10. Bačić, Ţeljko, T. Jogun, I. Majić, Tehnički vjesnik 25(1) 277-284 (2018)

11. Interactive IUPAC Compendium of Chemical Terminology (IUPAC Gold Book) http://goldbook.iupac.org/

12. R. Monoslk, M. Stred'ansky, E. Sturdik, 5(1) 109-120 (2012) doi:10.2478/ V10188012-0017-Z

13. J. Ali, J. Najeeb, M. A. Ali, M. F. Aslam, A. Raza., Bios. Bioelec. 8, 235 (2017) doi: $10.4172 / 2155-6210.1000235$

14. S. Malhotra, A. Verma, N. Tyagi, and V. Kumar, Int. J. Adv. Res. Innov. Id. Educ. 3(2) 3639-3644 (2017)

15. S. Sharma, H. Byrne, R. J. O'Kennedy, Essays Biochem. 60(1), 9-18 (2016) doi:10.1042/EBC20150002

16. G. Rocchitta, A. Spanu, S. Babudieri, et al., Sens. 16(6), 780 (2016) doi:10.3390/s16060780

17. Su Liang, Jia Wenzhao, Hou Changjun, Lei Yu, Rev. Bios. Bioelec. 26(5) 1788-1799 (2011) doi:10.1016/j.bios.2010.09.005.

18. P. Mehrotra, Oral. Biol. Craniofac. Res. 6(2), 153-159 (2016) doi: 10.1016/j.jobcr.2015.12.002

19. V. Kavita, Bioengin. Biomed. Sci. 7, 222 (2017) doi:10.4172/2155-9538.1000222

20. C., Tamerler, M. Sarikaya, Phil. Trans. R. Soc. A. 367, 1705-1726 (2009)

21. C. C. Adley, M. P. Ryan, Bhunia, A.K., Kim, M.S., Taitt. High Through. Screen. Food Saf. Asses. Biosens. Techn. Hypersp. Imag. Pract. Applic. 315-326, 2014

22. E. B. Bahadır, M. K. Sezgintürk, Nanomed. Biotechn. 44(1), 248-262, (2016) DOI: 10.3109/21691401.2014.942456

23. G. Ertürk, B. Mattiasson, Sens. 17(2), 390 (2017) doi: 10.3390/s17020390

24. P. Damborský, J. Švitel, J. Katrlík, Ess. Biochem. 60, 91-100 (2016) doi:10.1042/EBC20150010.

25. K. Narsaiah, S. N. Jha, R. Bhardwaj et al. J. Food Sci. Technol. 49(4), 383-406 (2012) doi:10.1007/s13197-011-0437-6.

26. J. Arlett, E. B. Myers, M. Roukes, Nat. Nanotech. 6(4), 203-15 (2011) 10.1038/nnano.2011.44.

27. J. Tamayo, K. Priscila, R. José, P. Alvaro, Calleja, Mont. Chem. Soc. Rev. 42(3) (2012) doi:10.1039/c2cs35293a.

28. K. Ramanathan, B. Danielsson, Biosen. Bioelec. 16(6), 417-423 (2001) doi:10.1016/S0956-5663(01)00124-5.

29. K. Ramanathan, B. Danielsson, Biosens. Bioelec. 16(6), 417-23 (2001)

30. I. Petrova, V. Zaripova, Y. Lezhnina, I. Mitchenko and V. Sokolskiy, 6th International Conference on Information, Intelligence, Systems and Applications (IISA) 1-6 (2015) doi: 10.1109/IISA.2015.7388016

31. V. M. Zaripova, I.Y. Petrova, Commun. Comp. Inform. Sci. 754, 479-489 (2017) 